\title{
Transfer of the data preprocessing parameters and fore- casting models
}

\author{
Arthur Stepchenko ${ }^{1}{ }^{*}$, Jurij Chizhov ${ }^{2}$, Ludmila Aleksejeva ${ }^{3}$ \\ 1, 2, 3 Riga Technical University, Riga, Latvia \\ ${ }^{2}$ Ventspils University of Applied Sciences, Ventspils, Latvia
}

\author{
Keywords \\ Forecasting models \\ NDVI \\ Pixel grid
}

Received: 1 October 2018

Accepted: 5 November 2018

Published: 19 December 2018

\begin{abstract}
Analysis and forecasting of the life cycle of vegetation are essential in planning agricultural work and monitoring crops and forecasting their productivity. In practice, vegetation indices are often used that are calculated from the values of satellite image pixels like normalized difference vegetation index (NDVI). Forecasting this index in precision agriculture allows indicating problems related to agricultural crop growth in time and making timely decisions about necessary measures to fix these problems. In the paper transferring approach for data preprocessing parameters and forecasting model is developed that provides forecasting of other normalized vegetation index time series without new preprocessing and training if the Euclidean distance between the time series used in the training and the time series using the data processing parameters and the forecasting model is small enough.
\end{abstract}

(C) 2018 The Author(s). Published by TAF Publishing.

\section{INTRODUCTION}

Human activities affect ecosystems, including the natural vegetation cover. Vegetation cover change is important factors that affect ecosystem condition and function. A change of vegetation cover may have long term impact on sustainable food production, freshwater and forest resources, the climate and human welfare $[1,2]$.

Vegetation indices calculated from satellite images can be used for monitoring temporal changes associated with vegetation. Vegetation Indices (VIs) are combinations of Digital Numbers (DNs) or surface reflectance at two or more wavelengths designed to take out a particular property of vegetation. Each of the VIs is designed to emphasize a particular vegetation property. Analyzing vegetation using remotely sensed data requires knowledge of the structure and function of vegetation and its reflectance properties. This knowledge enables the linking of vegetative structures and their condition to their reflectance behavior in an ecological system of interest $[3,4]$.

The NDVI is developed for estimating vegetation cover from the reflective bands of satellite data. The NDVI is an indica- tor which quantifies the amount of green vegetation. Past studies have demonstrated the potential of using NDVI to study vegetation dynamics. The NDVI data layer is defined as:

$$
N D V I=\frac{N I R-R E D}{N I R+R E D}
$$

Where NIR is reflectance in the near infrared band of the electromagnetic spectrum and RED is reflectance in the red band of the electromagnetic spectrum.

Greener and dense vegetation have low red light reflectance and a high near infrared reflectance, and thus high NDVI values. The NDVI real values, by definition, would be between -1 and +1 , where increasing positive values indicate increasing green vegetation, but low positive values and negative values indicate non-vegetated surface features such as water, barren land, rock, ice, snow, clouds or artificial materials $[5,6]$. The NDVI also has the ability to reduce external noise factors such as topographical effects and sun-angle variations.

\footnotetext{
${ }^{*}$ Corresponding author: Arthur Stepchenko

†email: arturs.Stepcenko@edu.rtu.lv
} 


\section{LITERATURE REVIEW}

The analysis of forecasting studies $[7,8,9,10]$ of the NDVI time series is made in the paper and some shortcomings are found. The studies $[7,8,9]$ do not always achieve high forecasting accuracy, but it depends on various factors, e.g., the use of additional data. Several studies used additional input data such as data of temperature or rainfall data. Obtaining additional data may be difficult, because these data may not always be available for the specific pixel; more often these data are available for large areas (average values in the municipality, district, etc.). Additional data also may not be available for free.

In all examined studies little attention is paid to the data preprocessing phase; preprocessing methods such as feature selection and feature extraction are not used, which helps to prepare input data set for time series forecasting tasks so that forecasting accuracy is increased.

Box-Jenkins ARIMA model as well as linear regression analysis used to forecast NDVI time series in several studies, are linear forecasting methods and are not robust to noise, while NDVI time series are nonlinear and noisy [11]. Moreover, the identification of a suitable ARIMA model is a timeconsuming and resource-consuming procedure.

\section{DATA AND RESEARCH AREA}

The research area used in the paper is Ventspils municipality of the Republic of Latvia. Data set consists of 814 smoothed NDVI images obtained from Moderate Resolution Imaging Spectroradiometer (MODIS) Terra satellite images with $250 \mathrm{~m}$ spatial resolution, the temporal resolution of 7 days and 16-bit radiometric resolution. These images cover the territory of Ventspils municipality. NDVI images were downloaded from Data service platform for MODIS Vegetation Indices time series processing at Vienna University of Natural Resources and Life Sciences. On the platform preprocessing of MODIS Terra NDVI images are made that includes smoothing pixel values and filling missing values due to cloud cover or bad weather conditions [12].

In the paper, nonlinear and nonstationary univariate NDVI time series with elements of additive noise and seasonal components are used [11]. Temporal resolution is 7 days. The number of observations for each NDVI time series is 814 and an interval of values $[-1 ; 1]$.

\section{VARIATIONAL MODE DECOMPOSITION}

In Variational Mode Decomposition (VMD) model, it is assumed that the real signal $f$ consists of several sub-signals uk, where $k=1, . ., K$. A sub-signal or intrinsic mode function is an Amplitude and Frequency Modulated (AM-FM) signal
[9] and it can be described with Expression 2:

$$
u_{k}(t)=A_{k}(t) \cos \left(\phi_{k}(t)\right)
$$

where $k$ is the sub-signal number, $K$ is the number of subsignals, $A_{k}(t)$ is $k$-th sub-signal amplitude and $\phi_{k}(t)$ is $k$-th sub-signal phase.

To calculate sub-signals, it is necessary to minimize the sum of $K$ sub-signal frequency bandwidth, provided that the sum of all $K$ sub-signals is equal to the original signal [7]. The unknowns are $K$ sub-signal center frequencies and $K$ intrinsic mode functions that are centered on these frequencies.

To obtain the correct results in both signal endpoints, using the algorithm of variational mode decomposition, the original signal is expanded, using "mirroring" [13]. The expanded signal is twice as long as the original signal, and its length is $T$. The Fourier transform is obtained from the extended signal, and all calculations are happening in the frequency domain. When all sub-signals in the frequency domain are obtained, then using inverse Fourier transform, the sub-signals in the time domain are obtained.

\section{PHASE SPACE RECONSTRUCTION WITH TIME DELAY METHOD}

The phase space is an abstract space, which describes a set of possible states of the system, where each possible state corresponding to the point in phase space [14].

For a chaotic time series, a phase space reconstruction with a time delay method according to Taken's theorem [15] is used. Using the Taken's theorem, a chaotic and dynamic system can be described with a set of delayed vectors. Using the phase space reconstruction method with a time delay, it is necessary to find two parameters-the suitable values of the embedding dimension $\mathrm{m}$ and time delay $\tau$. The accuracy of the time series prediction depends on these parameters.

\section{MODIFICATION OF VMD METHOD AND THE SUB-SIGNAL APPROXIMATION APPROACH}

The original VMD method uses a "mirroring" algorithm [13]. Variational mode decomposition method works with a signal whose length is $T$. However, from the perspective of time series forecasting, this "mirroring" approach causes certain problems - extended parts of time series gives investment in sub-signal computing process, and hence affects the subsignal values. It leads to a problem, that they can forecast with high accuracy only historical observations of time series when developing forecasting models.

To solve the problem caused by "mirroring", one of the options is not to use the extension of time series but twice cut the number of original signal observations received by the 
VMD method at the input. If the all original time series (that is used as extended time series) is with the number of observations $T$, then the middle part of time series contains $\mathrm{N}$ observations, and obtained sub-signal is corresponding to those $\mathrm{N}$ observations. Now, the real observations of the original time series will be replacing the extensions, and sub-signal values will contain information about the true value of input time series in the next time steps.

Accordingly, the extension at the beginning of the time series will include observations from the first observation to the point obtained by the formula 3 :

$$
A=\frac{T}{4}+1
$$

The extension at the end of the time series corresponds to the observations of the original time series from point $\mathrm{C}$ to the last observation. The endpoint of the new "original" NDVI time series $C$ is obtained by calculating the formula 4 :

$$
C=3 \frac{T}{4}+1
$$

The calculated proportionality of "original" (fragment AC) and "extended" time series correspond to a proportionality of original and extended time series obtained using the original VMD method. In other words, the original times series length is half of the extended time series length, besides the extended values is located at both endpoints of the original time series.

However, this modification is not yet applicable for forecasting new values of the NDVI time series, because the obtained sub-signal values are available only for the middle part of the input time series.

Accordingly, the time series value, that can be forecasted one time-step, will be located behind these middle part observations, in the time step $t=N+1$, while in the NDVI forecasting task need to forecast value in time step $t=T+$ 1. Therefore it is necessary to obtain the sub-signal values for all time steps. If the data set used in approximation algorithm consists of the sub-signal $u$, whose number of observations is $N$, then by submitting this sub-signal as a vector to the linear kernel function with a constant $\mathrm{c}=0$, is obtained matrix $N x N$, and it is describes with an Equation 5:

$$
K(u, u)=\left[\begin{array}{cccc}
u(1) u(1) & u(1) u(2) & \ldots & u(1) u(N) \\
u(2) u(1) & u(2) u(2) & \ldots & u(2) u(N) \\
\ldots & \ldots & \ldots & \\
u(N) u(1) & u(N) u(2) & \ldots & u(N) u(N)
\end{array}\right]
$$

Solving the homogenous linear equations system with the Singular Value Decomposition (SVD) method, whereas the coefficient matrix is used the matrix 5 , is obtained the null space $\mathrm{x}$ that describes the matrix 6 :

$$
x=\left[\begin{array}{ccccc}
x_{1}(1) & x_{1}(2) & x_{1}(3) & \ldots & x_{1}(N) \\
x_{2}(1) & x_{2}(2) & x_{2}(3) & \ldots & x_{2}(N) \\
\ldots & \ldots & \ldots & \ldots & \\
x_{N-1}(1) & x_{N-1}(2) & x_{N-1}(3) & \ldots & x_{N-1}(N)
\end{array}\right]
$$

Matrix 6 consisting of $\mathrm{N}$-1 linearly independent solutions and $N$ variables. Now can overwrite the linear kernel funca data set of two vectors: the NDVI time series y with $N$ observations and the sub-signal $\mathrm{u}$, as shown in matrix 7 : tion 5 as a linear kernel function, which at the input receives

$$
K([y u],[y u])=\left[\begin{array}{cc}
y(1) y(1)+u(1) u(1) & \ldots y(1) y(N)+u(1) u(N) \\
y(2) y(1)+u(2) u(1) & \ldots y(2) y(N)+u(2) u(N) \\
\ldots & \ldots \\
y(N) y(1)+u(N) u(1) & \ldots y(N) y(N)+u(N) u(N)
\end{array}\right]
$$

The null space (6) has one solution less than the number of unknowns. It is necessary to obtain one more equation - to find the particular solution $w 1$ of the first non-homogenous linear equations system, whereas the coefficient matrix A1 is used matrix 8 . 


$$
A=\left[\begin{array}{cc}
1 y(1) y(1)+u(1) u(1) & \ldots y(1) y(N-2)+u(1) u(N-2) \\
1 y(2) y(1)+u(2) u(1) & \ldots y(2) y(N-2)+u(2) u(N-2) \\
\ldots & \ldots \\
1 y(N) y(1)+u(N) u(1) & \ldots y(N) y(N-2)+u(N) u(N-2)
\end{array}\right]
$$

For depending parameter time series $y$, multiplied by the scaling factor coef is used. The value of the scaling factor is searched in the interval $[0,95 ; 1]$ with a step 0.000001 . By multiply matrix A1 with the solution w1 is obtained $\hat{y} 1$.
By adding the obtained solution vector $\mathrm{w} 1$ to the null space solutions, the coefficient matrix A2 is obtained as shown on the Equation 9:

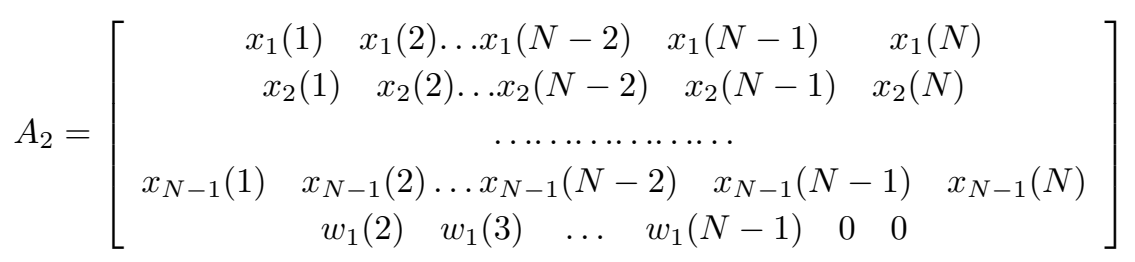

The vector of constant terms $\mathrm{b}$ used in the second nonhomogenous LES is also composed of $N$ elements. The el- ements of the vector bt(i) in the time step $t$ are obtained using the Equation System 10.

$$
b_{t}(i)=\left[\begin{array}{c}
y(t) y(1) x_{1}(1)+y(t) y(2) x_{1}(2)+\ldots+y(t) y(N) x_{1}(N) \\
y(t) y(\mathrm{D}) x_{2}(1)+y(t) y(2) x_{2}(2)+\ldots+y(t) y(N) x_{2}(N) \\
\ldots \ldots \ldots \ldots \ldots \ldots \ldots \\
y(t) y(1) x_{N-1}(1)+y(t) y(2) x_{N-1}(2)+\ldots+y(t) y(N) x_{N-1}(N)
\end{array}\right]
$$

First of all the sub-signal $u$ is initially approximated using linear regression analysis by least squares method. As the coefficient matrix is used reconstrued phase space from the NDVI time series. Dimension $m$ is taken from one to value given by the formula 3. As the appropriate dimension is taken value where RMSE between the sub-signal and initially approximated sub-signal is minimum. Now the coefficient matrix 8 is re-calculated using initially approximated sub-signal $\hat{u} 11$ and used in linear regression analysis whereas depending parameter is used $\hat{y} 1$. The solution is obtained using the least squares method. So the unknown vector $\hat{y} 1$ is approximated by the known vector $\hat{y} 2$. The vector element $b_{t}(i)$, where $i=N$ is calculated by Equation 11 :

$$
b_{t}(i)=\hat{y}_{2}(t)-w_{1}(1)
$$

Thus, by solving the second non-homogenous linear equation system in every time step $t$, where coefficient matrix A2 is obtained by Equation 9 and the vector of constant terms $\mathrm{b}$ after Equation system 10 and Equation 11, the particular solution wt of the second non-homogenous linear equation system is obtained from Expression 12:

$$
w_{t}=A_{2}^{-1} b_{t}
$$

where $A_{2}^{-1}$ is inverse matrix of the coefficient matrix A2. To obtain sub-signal $\mathrm{u}$ approximated value in the time step $t$, first the Equation ?? is used, which allows obtaining $N$ different $u_{i}(t)$ values, where $i=1, \ldots, N$ :

$$
\hat{u}_{i}(t)=\frac{\left(w_{t}(i)-y(t) y(i)\right)}{u(i)}
$$

And by all $i$ is calculated the mean value after the Equation 14:

$$
\hat{u}(t)=\frac{\sum_{i=1}^{N} \hat{u}_{i}(t)}{N}
$$

The result obtained by Equation 14) is an approximated value of sub-signal $u$ in time step $t$. Then corrections are used at each time step $t$. A suitable value of approximated subsignal in time step $\mathrm{t}$ is found by placing values from interval $[\hat{u}(t)-\operatorname{Max} A E ; \hat{u}(t)+\operatorname{Max} A E]$ in a linear regression model, where MaxAE is the maximum absolute error between observed and approximated subsignal $u$, and predicting the value of the NDVI (a certain value from historical data at certain time step). As suitable value $\hat{u}(t)$ is taken value that for corresponding linear regression model give minimal RMSE value between observed and predicted historical NDVI value. 


\section{STEPWISE REGRESSION ANALYSIS}

Stepwise regression analysis is a systematic method for sequent feature selection, where features are added to the multi-linear model and removed from it as a result of automatic procedures, based on the statistical significance of the feature in the regression analysis [16].

The original stepwise regression model does not include any feature. Then in each step is calculated $F$-statistic $p$-value, to test models with certain features [17]. The feature with the smallest $p$-value is added to the model, if it does not exceed the specified addition threshold and if the null hypothesis is rejected, that if add this feature to a model, it might have a zero coefficient. From features, that are already in the model, the feature with the highest $p$-value is removed from the model, if feature $p$-value exceeds the specific removal threshold, and if the null hypothesis is not rejected, that this feature has a zero coefficient.

\section{PRINCIPAL COMPONENT ANALYSIS}

Principal Component Analysis (PCA) is a statistical feature extraction method, which is using the orthogonal transformation to transform a potentially correlating data set to linearly uncorrelated data set, where data set features are called principal components [18]. PCA algorithm consists of finding the linear transformation of the original feature set $x 1, x 2, \ldots, x m$. Here, $m$ is the dimensionality of original feature set.

\section{LAYER RECURRENT NEURAL NETWORK}

Artificial Neural Networks (ANN) is a form of artificial intelligence, which tries to imitate the function of biological neurons that work on the human brain [19]. One of the most common artificial neural network models is a multilayer perceptron, which includes an input layer, output layer and one or more hidden layers [19]. In the case of the prediction task addressed in this paper, the output layer contains one neuron that gives the forecasted value of the time series. Each neuron in every layer receives the weighted inputs from the previous layer, and these weighted inputs are summed, using the combination function; this result of summing is fed as an argument to activation function, where this function value isthe output of the neuron, and it is fed to the next layer [19].

Layer Recurrent Neural Network (LRNN) is one of the dynamic, recurrent neural network forms, which is created by adding the feedback connections from the hidden layer to the context layer in multi-layer perceptron $[20,21]$. The context layer stores the values of the hidden layer with a time delay, thus providing useful information about the pre- vious input vector, and it determines the main quality - sequence memorizing [22].

\section{TRANSFER OF THE DATA PREPROCESSING PARAMETERS AND FORECASTING MODELS}

Two experiments are carried out with transferring of the data preprocessing parameters and models. The aim of the first experiment is to explore the transfer of forecasting models to NDVI time series of neighboring pixels by forecasting without the new training, and without using the subsignal approximation approach.

- The phase space with a dimension $m=50$ and time delay $\tau=1$ is obtained from time series of normalized difference vegetation index and thus input data set is obtained. Input data set is divided into two parts: a set of attributes and forecasting parameter. Both the set of attributes and forecasting parameter is divided into three parts, providing training, validation and test data set.

- From each feature set, there are selected informative features, using stepwise regression analysis, and the linearly uncorrelated feature sets are obtained using principal component analysis.

- Preprocessed training and validation data set are passed in layer recurrent neural network input for training and to obtain forecasting model.

-When on randomly chosen NDVI time series data preprocessing and training is performed, all the necessary data preprocessing parameters are saved, and a suitable forecasting model is obtained.

- Around this corresponding pixel of NDVI time series in satellite image, a grid is drawn that corresponds to the radius . Thus, around the chosen corresponding pixel of NDVI time series a large grid of size $11 \times 11$ is drawn, where there are 120 pixels excluding the trained central pixel.

- For each of these neighboring pixels, 120 NDVI time series are obtained and each of these time series first is preprocessed and forecasted using from the training time series obtained data preprocessing parameters and forecasting model. The RMSE values are calculated.

- Then, on each of these time series are individually obtained data preprocessing parameters and the forecasting model, then forecasts and RMSE values are calculated.

For each time series, both RMSE values are compared. The experiment is repeated 100 times, each time forecasting 120 time series. The aim of the second experiment is to explore the transfer of forecasting models to NDVI time series of neighboring pixels by forecasting without the new training and using the sub-signal approximation approach.

- Using developed modification of the VMD method with 
from this method obtained the sub-signal value approximation approach, an appropriate sub-signal is obtained for normalized difference vegetation index. A phase space with $m=50$ and time delay $\tau=1$ is reconstructed for sub-signal as well as phase space is reconstructed with the same parameters for the NDVI time series.

- Phase spaces are combined to produce input data set that is divided into two parts: the attribute set and the forecasting parameter. Both the attribute set and forecasting parameter are divided into three parts, providing training validation and test data set.

- From each feature set, there are selected informative features, using stepwise regression analysis, and linearly uncorrelated feature sets are obtained, using principal component analysis.

- Preprocessed training and validation data set are passed in layer recurrent neural network input for training and to obtain forecasting model.

- The $11 \times 11$ pixel grid is obtained from the satellite image (121 pixels in total), where the central pixel is the pixel on which NDVI time series preprocessing and training are performed.

- Each of the 120 time series is preprocessed and predicted, first using the transmitted parameters and models derived from the central pixel NDVI time series, and then using the individual parameters and models obtained on each time series, in both cases calculating the RMSE values between the observed and the predicted NDVI time series.

The experiment is repeated 100 times each time forecasting 120 time series.

\section{EXPERIMENTAL RESULTS}

In fifteen cases of the first experiment obtained results are shown in Figure 1. On the binary image (Figure 1), with the yellow color (the value is one) are marked those pixels on which time series can use transferred data preprocessing parameters and forecasting model. With the blue color (the value is zero) are marked those pixels on which time series is necessary to use the individually obtained data preprocessing parameters and original forecasting model.

On Figure 2, a Euclidean distance with a threshold 0.8 is shown.

Euclidean distances are calculated between the central pixel time series and all other time series. Analyzing Figure 3, it is concluded that transferring preprocessing parameters and forecasting models and, performing the forecasting, a similar RMSE value can be obtained, if between in training used time series and time series where there are used transferred parameters and models, the Euclidean distance is equal or lower than the value of threshold 0.8 .

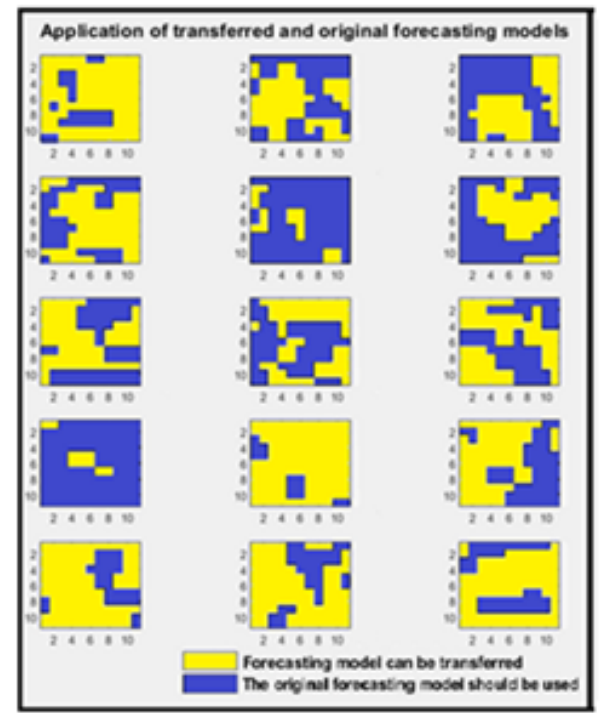

Fig. 1. Application of the forecasting model without approximation approach

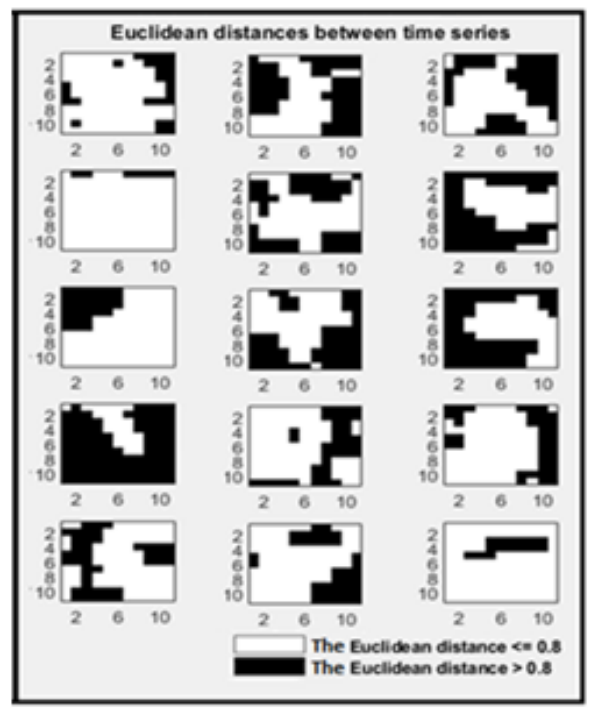

Fig. 2. Euclidean distances between time series with a threshold 0.8

In fifteen cases of the second experiments obtained results are shown in Figure 3. Figure 3 is a binary image where with yellow color is marked those pixels to which time series can transfer data preprocessing parameters and forecasting model, but with blue color are marked those pixels on which time series transfer is not possible to perform.

On Figure 4, a Euclidean distance with a threshold 0.1 is shown.

The forecasting accuracy in many cases is similar to the results provided by a trained forecasting model for a specific time series of normalized difference vegetation index. 


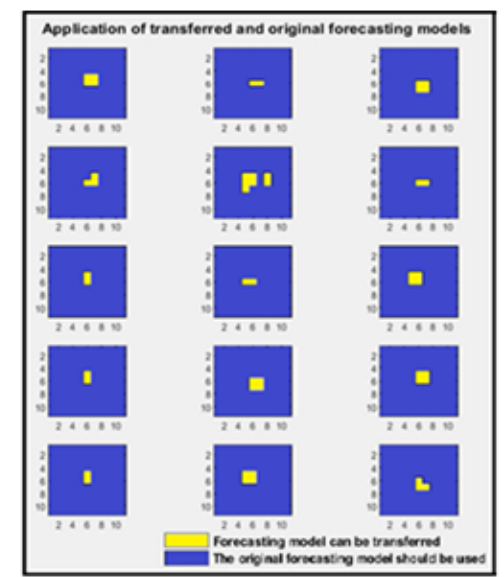

Fig. 3. Application of the forecasting model with approximation approach

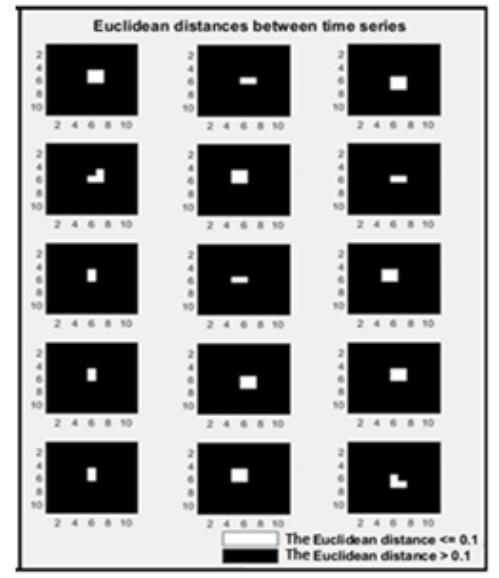

Fig. 4. Euclidean distances between time series with a threshold 0.1

\section{CONCLUSION}

By forecasting the NDVI time series, the data preprocessing parameters and forecasting model can be obtained which can be used to forecast other NDVI time series with similar accuracy compared to the accuracy that can be achieved by forecasting the time series with individually obtained data preprocessing parameters and forecasting model, if Euclidean distance between both the time series is less or equal than the defined threshold.

The combination of methods used in this paper without ap- proximation approach can be used, if it is necessary to forecast values of the time series of normalized difference vegetation index for the large area in a relatively short period and where a slight decrease in forecasting accuracy is allowed.

The combination of methods used in this paper with the approximation approach can be used, when forecasts of the time series of NDVI with high accuracy are needed, but for the relatively small area.

\section{REFERENCES}

[1] M. Badamasi, S. Yelwa, M. AbdulRahim, and S. Noma, "NDVI threshold classification and change detection of vegetation cover at the falgore game reserve in Kano State, Nigeria," Sokoto Journal of the Social Sciences, vol. 2, no. 2, pp. 174-194, 2010.

[2] C. L. S. Tablatin, F. F. Patacsil, and P. V. Cenas, "Design and development of an information technology fundamentals multimedia courseware for dynamic learning environment," Journal of Advances in Technology and Engineering Studies, vol. 2, no. 5, pp. 202-210, 2016. doi: https://doi.org/10.20474/jater-2.6.5 
[3] N. B. Duy, T. T. H. Giang, and T. S. Son, "Study on vegetation indices selection and changing detection thresholds selection in land cover change detection assessment using change vector analysis," in International Environmental Modelling and Software Society, Jiangsu, China, 2012.

[4] K. M. Attom, M. and N. Al-Akhras, "Geo environmental utilization of iron-filing with cement in soil stabilization," International Journal of Technology and Engineering Studies, vol. 2, no. 2, pp. 32-37, 2016. doi: https://doi.org/10.20469/ ijtes.2.40001-2

[5] E. Sahebjalal and K. Dashtekian, "Analysis of land use-land covers changes using Normalized Difference Vegetation Index (NDVI) differencing and classification methods," African Journal of Agricultural Research, vol. 8, no. 37, pp. 4614-4622, 2013. doi: https://doi.org/10.5897/ajar11.1825

[6] R. Suryanita, H. Maizir, and H. Jingga, "Prediction of structural response based on ground acceleration using artificial neural networks," International Journal of Technology and Engineering Studies, vol. 3, no. 2, pp. 74-83, 2017. doi: https: //doi.org/10.20469/ijtes.3.40005-2

[7] L. Ji and A. J. Peters, "Forecasting vegetation greenness with satellite and climate data," IEEE Geoscience and Remote Sensing Letters, vol. 1, no. 1, pp. 3-6, 2004. doi: https://doi.org/10.1109/lgrs.2003.821264

[8] A. Asoka and V. Mishra, "Prediction of vegetation anomalies to improve food security and water management in India," Geophysical Research Letters, vol. 42, no. 13, pp. 5290-5298, 2015. doi: https://doi.org/10.1002/2015gl063991

[9] N. Clements, S. Sarkar, and W. Wei, "Multiplicative spatio-temporal models for remotely sensed normalized difference vegetation index data," Journal of International Energy Policy, vol. 3, no. 1, pp. 1-16, 2014. doi: https://doi.org/10. 19030/jiep.v3i1.8941

[10] Y. X. Lee and Z. W. Zhong, "A study of the relationship between adverse weather conditions and flight delay," Journal of Advances in Technology and Engineering Research, vol. 2, no. 4, pp. 113-117, 2016. doi: https://doi.org/10.20474/ jater-2.4.2

[11] J. R. Jensen and K. Lulla, Introductory digital image processing: A remote sensing perspective. New York, NY: Taylor \& Francis, 1987.

[12] F. Vuolo, M. Mattiuzzi, A. Klisch, and C. Atzberger, "Data service platform for MODIS vegetation indices time series processing at BOKU Vienna: Current status and future perspectives," in Earth Resources and Environmental Remote Sensing/GIS Applications III, California, CA. International Society for Optics and Photonics, 2012.

[13] K. Dragomiretskiy and D. Zosso, "Variational mode decomposition," IEEE Transactions on Signal Processing, vol. 62, no. 3, pp. 531-544, 2013. doi: https://doi.org/10.1109/tsp.2013.2288675

[14] G. Chakrabarti and C. Sen, Anatomy of global stock market crashes: An empirical analysis. New York, NY: Springer Science \& Business Media, 2012.

[15] A. A. Bell, C. Seiler, J. N. Kaftan, and T. Aach, "Noise in high dynamic range imaging," in Proceeding of 15th IEEE International Conference on Image Processing, San Diego, CA, 2008.

[16] M. Templ, A. Kowarik, and P. Filzmoser, "Iterative stepwise regression imputation using standard and robust methods," Computational Statistics \& Data Analysis, vol. 55, no. 10, pp. 2793-2806, 2011.

[17] Z. M. Yusof, S. Abdullah, and S. S. S. Yahaya, “Comparing the performance of modified ft statistic with ANOVA and kruskal wallis test," Applied Mathematic Journal, vol. 7, no. 2L, pp. 403-408, 2013. doi: https://doi.org/10.12785/amis/072104

[18] J. Mohamad-Saleh, B. S. Hoyle et al., "Improved neural network performance using principal component analysis on matlab," International Journal of the Computer, the Internet and Management, vol. 16, no. 2, pp. 1-8, 2008.

[19] A. Shabri and R. Samsudin, "Daily crude oil price forecasting using hybridizing wavelet and artificial neural network model," Mathematical Problems in Engineering, vol. 1, no. 2, pp. 1-10, 2014. doi: https://doi.org/10.1155/2014/ 201402

[20] M. Y. El-Sharkh and M. Rahman, "Forecasting electricity demand using dynamic artificial neural network model," in International Conference on Industrial Engineering and Operations Management, California, CA, 2012.

[21] F. H. Nordin, F. H. Nagi, and A. A. Z. Abidin, "Comparison study of computational parameter values between LRN and NARX in identifying nonlinear systems," Turkish Journal of Electrical Engineering \& Computer Sciences, vol. 21, no. 4, pp. 1151-1165, 2013. doi: https://doi.org/10.1109/iccas.2008.4694674

[22] J. L. Elman, "Finding structure in time," Cognitive Science, vol. 14, no. 2, pp. 179-211, 1990. doi: https://doi.org/10. 1016/0364-0213(90)90002-e 